Document downloaded from:

http://hdl.handle.net/10251/121398

This paper must be cited as:

Benítez-González, J.; Mora Almerich, J. (2018). Advanced RF Interferometry Structure for Improving Operation Range. IEEE Photonics Technology Letters. 30(18):1637-1640. https://doi.org/10.1109/LPT.2018.2865005

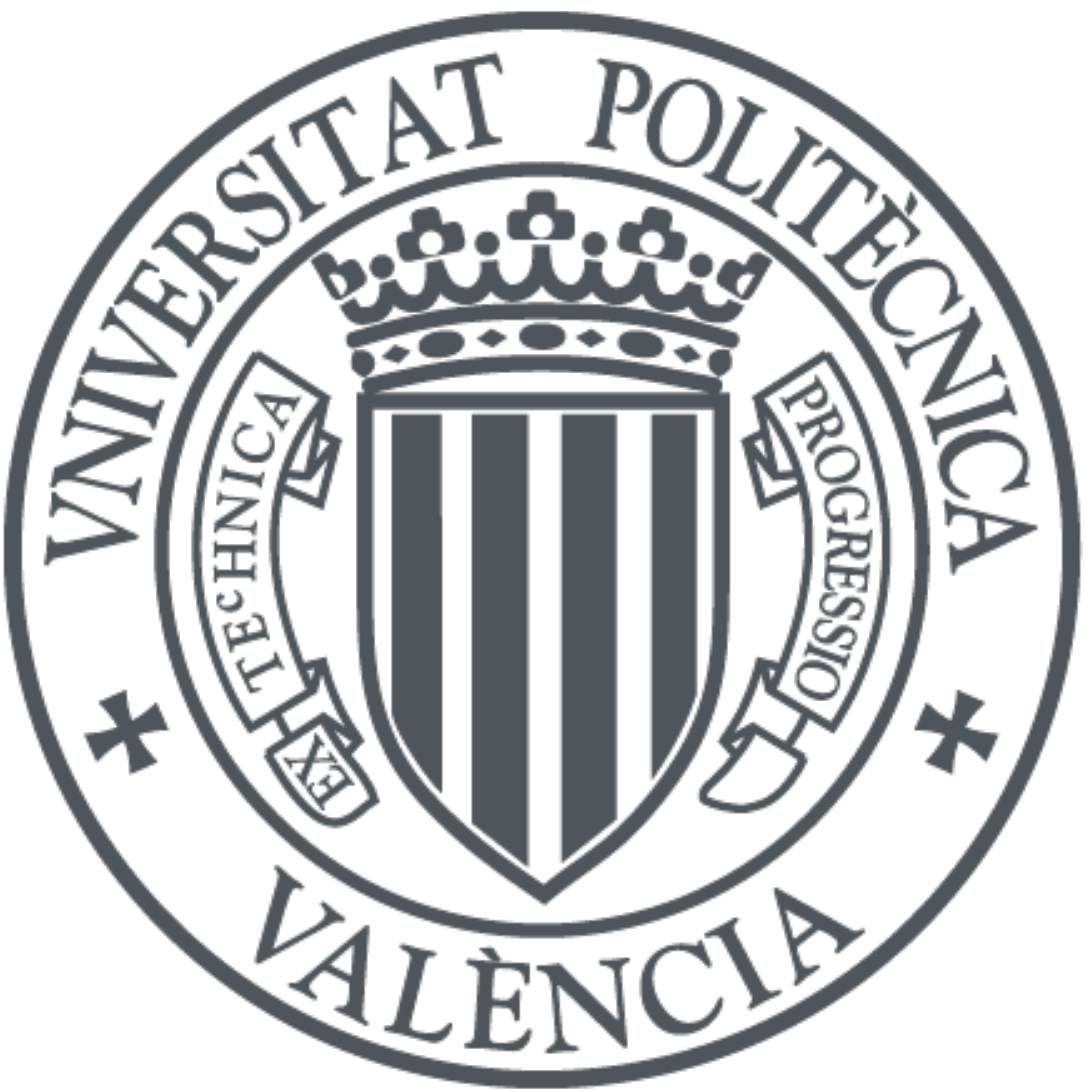

The final publication is available at

http://doi.org/10.1109/LPT.2018.2865005

Copyright Institute of Electrical and Electronics Engineers

Additional Information 


\title{
Advanced RF interferometry structure for improving operation range
}

\author{
J. Benítez and J. Mora
}

\begin{abstract}
In this work, a novel structure combining low coherence interferometry (LCI) and microwave photonics (MWP) is proposed. In this case, the electro-optic modulator (EOM) is strategically placed inside the interferometric structure in order to modify the behavior of the whole MWP-LCI system. Specifically, limiting effects which are inherently generated as the DC term, the self-reflection term and the carrier suppression effect (CSE) are avoided. This fact enables the improvement of the MWP-LCI operating range in comparison to previous proposals. Moreover, a detailed description of the MWP-LCI structure are addressed in this work to support by the corresponding experimental demonstration and theoretical development. In addition, typical LCI capabilities such as penetration depth, resolution and sensitivity are also measured. For the MWP-LCI structure proposed here, a $1 \mathrm{~cm}$ penetration depth with a resolution of $120 \mu \mathrm{m}$ and a sensitivity beyond $50 \mathrm{~dB}$ are achieved.
\end{abstract}

Index Terms-Low coherence interferometry, microwave photonics, optical path difference, electrical transfer function.

\section{INTRODUCTION}

$\mathrm{O}$ PTICAL low coherence interferometry (LCI) constitutes an excellent optical measurement technique able to provide an axial positioning precision close to the order of the microns [1]. This technique is based on the analysis of the interference pattern generated by the light reflections in a reference surface (e.g. a mirror) and in the sample under study. In this process, a low coherence source is employed so the reflective events of the sample are temporally and spatially resolved. From the first reports [2], LCI has attracted the interest of many authors due to its applicability in a wide variety of areas. Although its main field of application is imaging where the optical coherence tomography (OCT) is contained [3], LCI has been also extended to applications such as: components characterization [4], optical sensing [5] or art conservation [6].

Nowadays, the challenges of LCI basically consist on the improvement of its key parameters: penetration range, resolution and sensitivity. Moreover, the rapid application development with different requirements implies the search of a better stability in the optical interference pattern. Both facts are currently limited by the lack of solutions that merge

Manuscript received May 10th, 2018. The research leading to these results has received funding from the National Project TEC2014-60378 funded by the Ministerio de Ciencia y Tecnología and the regional project PROMETEO FASE II/2013/012 funded by the Generalitat Valenciana.

J. Benítez and J. Mora* are with the ITEAM Research Institute, Universitat Politècnica de València, Valencia, Spain (*e-mail: jmalmer@iteam.upv.es). compactness and cost-effectiveness. In this context, we propose the combination of the traditional LCI technique with the field of microwave photonics (MWP). MWP combines the radiofrequency $(\mathrm{RF})$ engineering and the optical technology [7]. In this sense, the inherent stability of the interference pattern generated in the electrical domain permits to explore innovative solutions to improve the current issues of traditional LCI.

Recently, several systems combining LCI and MWP have been reported in the literature [8-10]. These schemes use optical pulsed-light sources instead of broadband sources as shown in [11-13]. Since the LCI resolution is proportional to the bandwidth of the optical source, the employment of incoherent sources is a potential candidate against coherent sources, which are spectrally limited. These proposals are based on the retrieval of the optical path differences introduced by a sample through the electrical transfer function of the system [11-13]. In this way, previous contributions permit to establish an analogy between traditional LCI and MWP-LCI. Although these contributions constitute the first step to demonstrate the feasibility of MWP-LCI systems, several improvements are still necessary. Indeed, different degrading effects limit considerably the system operation range, also known as penetration depth. Typically, MWP-LCI systems have an inherent baseband (BB) contribution, which contains a DC term and a self-reflection term similar to conventional LCI architectures [11-13]. Moreover, the use of a dispersive element produces the carrier suppression effect (CSE) as usually observed in MWP schemes [11, 12]. In order to overcome these drawbacks, alternative proposals are found as the MWP-LCI structure by means of balanced photodetection [13]. However, recent solutions introduce more complexity to the MWP-LCI system by adding electrical and electro-optical components reducing the useful operation range.

In this sense, this work proposes a novel MWP-LCI structure that is able to highly remove the $\mathrm{BB}$ term and it is free from self-reflections [11-13]. Furthermore, the MWP-LCI system is also free from CSE $[11,12]$. All these improvements are addressed by strategically allocating the electro-optic modulator inside the interferometric structure. The principle of operation of this MWP-LCI structure is deeply detailed and the corresponding theoretical analysis is also addressed. In this sense, a multilayered sample is considered in the theoretical analysis in order to show the potentiality of our proposal. Finally, some LCI capabilities are experimentally 


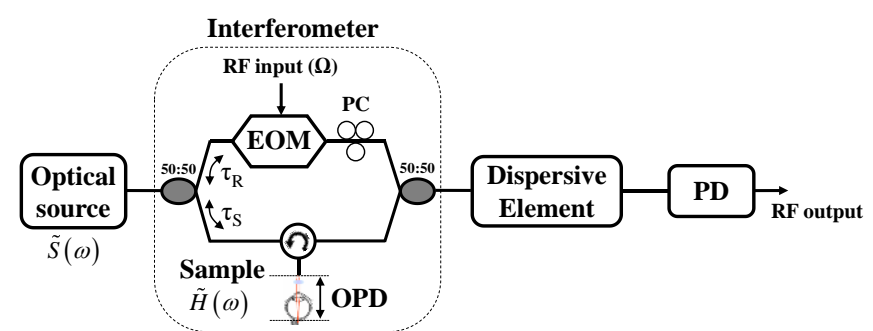

Fig. 1. Layout of the MWP-LCI structure with the electro-optic modulator in the upper branch of the interferometer.

demonstrated in this work. Specifically, a penetration depth of $1 \mathrm{~cm}$, an average system resolution of $120 \mu \mathrm{m}$ and a sensitivity beyond $50 \mathrm{~dB}$ are achieved. All experimental results are supported by the theoretical simulations.

\section{PRINCIPLE OF OPERATION}

The layout of the proposed MWP-LCI structure is shown in Fig. 1. Firstly, an incoherent optical source generates an optical signal which is described by $\mathrm{S}(\omega)$. This optical signal is split into two using a 50:50 fiber coupler and launched into a Mach-Zehnder interferometer. The upper branch is composed of an electro-optic modulator (EOM), which performs an amplitude modulation. The RF signal $(\Omega)$ employed in this process is provided by a vector network analyzer (VNA). The strategic placement of this EOM is the key element that modifies the response of the MWP-LCI system compared to previous proposals [11-13]. The sample under test, which is described by the optical transfer function $\mathrm{H}(\omega)$, is located in the lower branch of the interferometer. Experimentally, the sample role is achieved through a variable delay line (VDL). This device can be easily manipulated to introduce a given optical delay. Note that the optical delays produced in the upper $\left(\tau_{R}\right)$ and lower $\left(\tau_{S}\right)$ branches have to be exactly equal $\left(\tau_{\mathrm{R}}=\tau_{\mathrm{S}}\right)$ so the optical path difference between the arms of the interferometer would be exclusively introduced by the sample. Moreover, a polarization controller (PC) is also considered in order to control the interference pattern generated at the output of the 50:50 fiber coupler. Following, the optical signal is launched into a dispersive element, characterized by its accumulated chromatic dispersion parameter $\left(\varphi_{2}\right)$. Finally, a photodetector (PD) performs the optoelectronic conversion generating the RF output signal. To obtain the OPDs introduced by the sample, the electrical transfer function of the MWP-LCI structure is obtained and processed by means of a VNA.

In order to ease the comprehension of the MWP-LCI principle of operation, the multilayered sample is described as:

$$
\tilde{H}(\omega)=\sum_{n} H_{n} e^{-2 j \omega \tau_{n}}
$$

where $\mathrm{H}_{\mathrm{n}}$ and $\tau_{\mathrm{n}}$ represent the reflectivity and delay introduced by the nth-layer of the samplem respectively. Note that the factor 2 in the exponential considers the round-trip travel to each layer.

Moreover, the optical source distribution considered is defined through the next Fourier transform operation [12]:

$$
S(\Omega)=\frac{1}{2 \pi} \int_{-\infty}^{\infty} \tilde{S}(\omega) e^{j \frac{1}{2} \varphi_{2} \Omega \omega} d \omega
$$

Considering Eq. (1) and (2) and following a similar mathematical analysis as proposed in [14], the electrical transfer function of the MWP-LCI proposal is given by the next expression:

$$
\begin{aligned}
& I(\Omega) \propto \underbrace{\left(a_{0} a_{-1}^{*} e^{j \frac{1}{2} \varphi_{2} \Omega^{2}}+a_{1} a_{0}^{*} e^{-j \frac{1}{2} \varphi_{2} \Omega^{2}}\right) S(\Omega)}_{B B} \\
& -\underbrace{a_{-1}^{*} e^{j \frac{1}{2} \varphi_{2} \Omega^{2}} \sum_{n} H_{n} S\left(\Omega-\Omega_{n}\right)}_{L C I\left(+\Omega_{n}\right)}-\underbrace{a_{1} e^{-j \frac{1}{2} \varphi_{2} \Omega^{2}} \sum_{n} H_{n}^{*} S\left(\Omega+\Omega_{n}\right)}_{L C I\left(-\Omega_{n}\right)}
\end{aligned}
$$

where the different $a_{k}$ terms are related to the modulation format employed in the EOM [12].

From Eq. (3), we can distinguish two contributions. First, we observe a component labelled as BB, which is an inherent contribution to a conventional LCI system. The second contribution from Eq. (3) represents the LCI key information. This contribution produces an RF resonance in the electrical response for each OPD introduced by the sample. In this way, the measurement of the OPD is determined through the RF domain by means of the radiofrequency signal $\Omega$ [11-13].

In previous proposals [11-13], the $\mathrm{BB}$ component is dependent on the sample $H(\omega)$. This fact is the origin of a DC term and a self-reflection term in a multilayered sample scenario [13]. Unfortunately, the DC term limits the penetration depth for low values of the OPD while the selfreflection term generates residual contributions, which also reduce the useful operation range. Nevertheless, the BB component of our proposal is only proportional to the Fourier transform of the optical source spectrum $(\mathrm{S}(\Omega))$. Therefore, a DC term located around baseband is generated but the selfreflection term is avoided. In addition, Eq. (3) shows that different $\mathrm{a}_{\mathrm{k}}$ terms of the modulator are also multiplying the BB contribution. By controlling the modulation format employed in the EOM, we can easily modify the value of the $a_{k}$ terms and change the resultant electrical transfer function. In order to remove the DC term, we can configure the modulator with the coefficient $\mathrm{a}_{0}=0$ by means of a given bias voltage. In the MWP field, this means that we are generating a suppressed optical carrier modulation. Therefore, the DC term is eliminated in the electrical transfer function of Eq. (3) when $\mathrm{a}_{0}=0$ without affecting the main LCI contribution. Consequently, the useful operation range of the MWP-LCI structure is considerably improved by eliminating completely the BB contribution.

The second contribution from Eq. (3) has two terms: one related to $\mathrm{S}\left(\Omega+\Omega_{\mathrm{n}}\right)$ and another to $\mathrm{S}\left(\Omega-\Omega_{\mathrm{n}}\right)$, both proportional to $H(\omega)$. After the photodetection, this contribution produces an RF resonance for each OPD introduced by the sample. Indeed, the central frequency of each RF resonances $\left( \pm \Omega_{\mathrm{n}}\right)$ is closely related to the value of each OPD from the sample. The relationship between OPD and central frequency is ruled by 


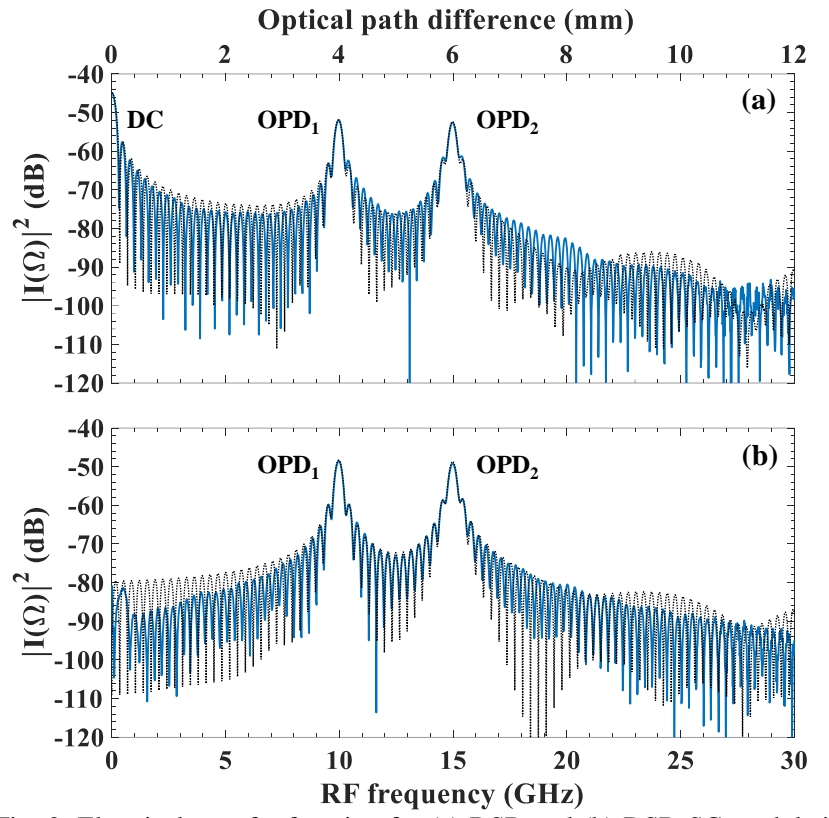

Fig. 2. Electrical transfer function for (a) DSB and (b) DSB-SC modulation format. Theoretical simulations are included in dashed black line and experimental results in solid blue line.

the dispersive element, demonstrating the key functionality of our approach:

$$
\Omega_{n}=2 \frac{O P D_{n}}{c_{0} \cdot\left|\varphi_{2}\right|}
$$

where $\mathrm{c}_{0}$ represents the speed of light in vacuum.

In order to visualize the principle of operation, Fig. 2 represents the electrical transfer function from Eq. (3). On one hand, Fig. 2(a) represents the electrical transfer function when the modulator performs a double sideband modulation (DSB) similar to previous proposals $[8,10]$. On the other hand, Fig. 2(b) considers a double sideband suppressed carrier (DSB-SC) modulation according to our approach. The modulator is biased at the minimum transmission point $\left(\mathrm{a}_{0}=0\right)$ to realize DSB-SC modulation with a bias voltage $\mathrm{V}_{\mathrm{DC}}=11.7 \mathrm{~V}$. Additionally, a square-shaped profile of $8.8 \mathrm{~nm}$ bandwidth is employed. A two-layered sample is considered with $\mathrm{H}_{1}=\mathrm{H}_{2}=1$ and $\tau_{1}=13 \mathrm{ps}$ and $\tau_{2}=20 \mathrm{ps}$. These delays produce an OPD given by: $\mathrm{OPD}_{\mathrm{n}}=\mathrm{c}_{0} \bullet \tau_{\mathrm{n}}(\mathrm{n}=1,2)$. Consequently, an $\mathrm{OPD}_{1}$ value is $3.9 \mathrm{~mm}$ and an $\mathrm{OPD}_{2}$ value is $5.9 \mathrm{~mm}$. Finally, a standard single mode fiber (SSMF) is considered as the dispersive element, with an accumulated chromatic dispersion $\left(\varphi_{2}\right)$ of $448 \mathrm{ps}^{2}$.

We observe in Fig. 2(a) that a DC term is generated close to low OPD values, which is typical for previous schemes based on amplitude modulation [11, 13]. However, our approach highly reduces the DC term $(\approx 35 \mathrm{~dB})$ due to the DSB-SC modulation. According to Eq. (4), two RF resonances centered at 10 and $15 \mathrm{GHz}$ are present due to the corresponding $\mathrm{OPD}_{1}$ and $\mathrm{OPD}_{2}$ values, respectively. However, self-reflection contributions are completely removed compared to previous MWP-LCI schemes with multilayered samples [10]. In this sense, the removal of the whole BB contribution allows a proper measurement of OPDs generated at very low values

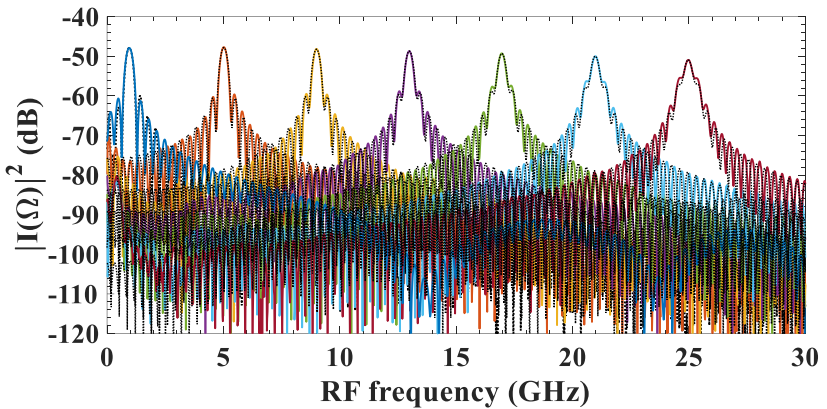

Fig. 3. Electrical transfer functions for suppressed carrier modulation $\left(a_{0}=0\right)$ when different OPDs are considered. Theoretical simulations are included in dashed black line and experimental results in solid color line.

extending the useful operation range. Note that Fig. 2 includes the theoretical simulation of Eq. (3), showing an excellent agreement with the experimental results.

Finally, we observe in Eq. (3) that the DSB-SC modulation $\left(a_{0}=0\right)$ permits to highly reduce the baseband contribution, which the CSE term is included. However, the structure achieves a LCI term free from CSE compared to previous schemes [11, 12], independently from the modulation format (DSB or DSB-SC). Indeed, the CSE is only present in the DC term as shown in Eq. (3). Therefore, Fig. 2(a) and Fig. 2(b) show the removal of the BB contribution for DSB-SC with similar response for the LCI contribution compared to DSB modulation. Therefore, the strategic placement of the EOM inside the interferometric structure improves the operation range of the MWP-LCI structure as the CSE is avoided in the LCI contribution.

\section{MWP-LCI CAPABILITIES}

In this section, the MWP-LCI structure presented in Fig. 1 is characterized and different LCI capabilities such as penetration depth, resolution and sensitivity are measured. All the experimental results consider a square-shaped profile of $8.8 \mathrm{~nm}$ and a SSMF as dispersive element.

Firstly, different OPDs are set in the interferometer to measure the electrical transfer functions in the considered range. The experimental results are depicted in Fig. 3 with OPD values up to $10 \mathrm{~mm}$. According to the discussion of Eq. (3), different $\mathrm{RF}$ resonances are generated up to $25 \mathrm{GHz}$ with an efficient reduction of $\mathrm{BB}$ contribution. In addition, no fading due to CSE is observed over the electrical response since the LCI contribution is free from this effect in our proposal. Both facts imply that any OPD can be correctly measured through the analysis of the RF resonances, improving the operation range compared to previous proposals $[11,12]$. The theoretical simulation of Eq. (3) is also added to Fig. 3, showing good agreement with the experimental results.

Following, the central frequency of each RF resonance is precisely measured for each OPD value as depicted in Fig. 4(a). In this case, two different dispersive elements are considered with a dispersion of $-222 \mathrm{ps}^{2}(\square)$ and $-448 \mathrm{ps}^{2}(\bullet)$. As predicted by Eq. (4), a linear relationship is found between the OPD and the central frequency, with slopes of $4.62(\square)$ and $2.41 \mathrm{GHz} / \mathrm{mm}(\bullet)$. In the measured range, a penetration depth of $1 \mathrm{~cm}$ is experimentally achieved. Note that higher 


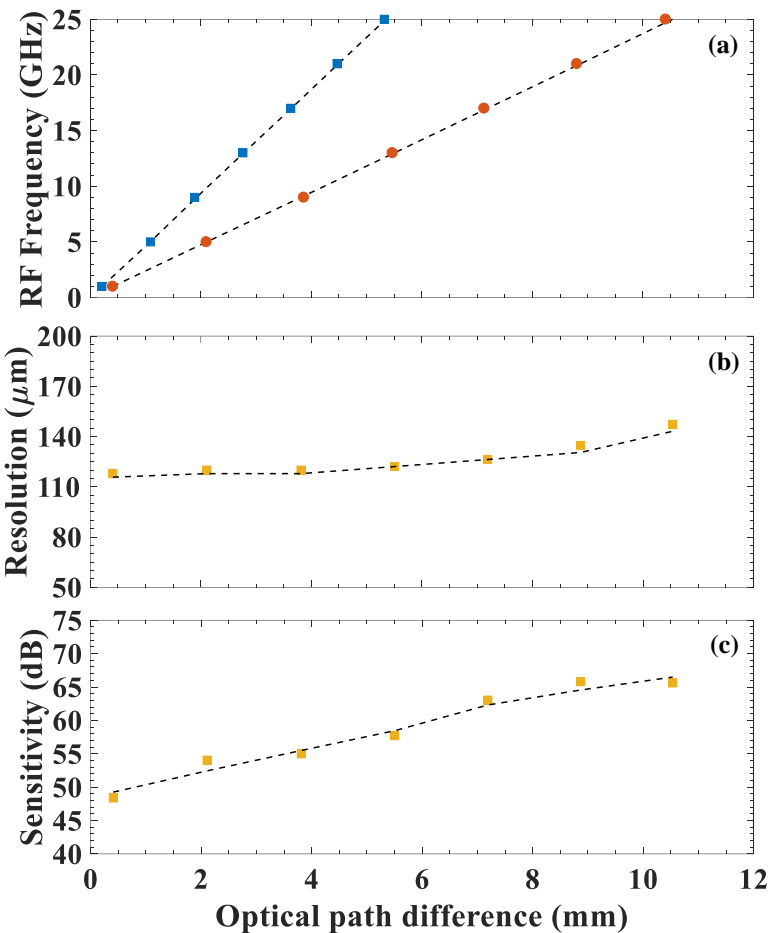

Fig. 4. (a) Central frequency of the RF resonance vs OPD when a $-222 \mathrm{ps}^{2}$ $(\square)$ and a $-448 \mathrm{ps}^{2}(\bullet)$ dispersive elements are considered. (b) Resolution vs OPD. (c) Sensitivity vs OPD. Theoretical predictions are plotted in dashed black line and experimental results are represented as dots.

penetration depth can be obtained by increasing the value of the dispersion $\varphi_{2}$.

The system resolution is also depicted in Fig. 4(b). In MWP-LCI, the resolution $(\delta \mathrm{z})$ is determined by the $3 \mathrm{~dB}$ bandwidth of the RF resonance [11]. The experimental results of Fig. 4(b) show an average resolution of $120 \mu \mathrm{m}$. For higher OPDs, a slight loss of resolution is observable due to the thirdorder dispersion from the dispersive element.

Finally, the sensitivity of the system is analyzed in Fig. 4(c). In traditional LCI, sensitivity is generally defined as the lower reflectivity coming from the sample that can be detected by the system [3]. Experimentally, the difference between the noise contribution when the sample is eliminated and the peak amplitude of each RF resonance is considered. In this case, the sensitivity varies from 50 to $65 \mathrm{~dB}$ due to the noise contribution as plotted in Fig. 4(c). For low OPDs, the noise is determined by the non-ideal generation of the suppressed carrier modulation. This implies that the DC term is highly removed, but not completely. For higher OPDs, the noise contribution is induced by the floor noise of the VNA employed in the measurement.

\section{CONCLUSION}

In conclusion, a novel MWP-LCI structure is proposed in this work with a strategical placement of the EOM inside of the interferometric structure. The location of the EOM and the generation of a DSB-SC modulation permits to remove the DC term and the self-reflection terms observed in previous proposals. Both terms are intrinsically found in LCI systems. Moreover, this structure is free from CSE which is a drawback in MWP architectures when amplitude modulators are considered. As consequence, the operation range of the system is highly improved in our MWP-LCI proposal. The possibility of retrieving the information of the sample through the analysis of the electrical transfer function is demonstrated in the considered whole range. A penetration depth of $1 \mathrm{~cm}$, an average resolution of 120 and a sensitivity beyond $50 \mathrm{~dB}$ are experimentally demonstrated.

\section{REFERENCES}

[1] J. Pluciński, R. Hypszer, P. Wierzba, M. Strąkowski, M. JędrzejewskaSzczerska, M. Maciejewski and B.B. Kosmowski, "Optical lowcoherence interferometry for selected technical applications," Bull. Pol. Acad. Sci., Tech. Sci., vol. 56, no. 2, pp. 155-172, 2008.

[2] A. F. Fercher, K. Mengedoht, and W. Werner, "Eye-length measurement by interferometry with partially coherent light," Opt. Lett., vol. 13, no. 3, pp. 186-188, 1988.

[3] M. Wojtkowski, "High-speed optical coherence tomography: Basics and applications," Appl. Opt., vol. 49, no. 16, pp. D30-D61, 2010.

[4] W. K. Liu, C. Y. Chen, C. Wei, and Y. J. Chen, "Improved technique for the characterization of micro-ring resonator using low coherence measurement," Opt. Lett., vol. 40, no. 12, pp. 2909-2912, 2015.

[5] K. Liu, D. Ju, S. Wang, J. Jiang, M. Xiao, X. Wang, T. Liu, "An Improved Optical Fiber Remote Sensing Method Based on Polarized Low-Coherence Interferometry," IEEE Photon. J., vol. 10, no. 1, pp. 19, 2018.

[6] P. Targowski and M. Iwanicka, "Optical Coherence Tomography: its role in the non-invasive structural examination and conservation of cultural heritage objects-a review," Appl. Phys. A, vol. 106, no. 2, pp. 265-277 2012.

[7] J. Capmany and D. Novak, "Microwave Photonics combines two worlds," Nat. Photonics, vol. 1, no. 6, pp. 319-329, 2007.

[8] T. J. Ahn, Y. Park and J. Azana, "Ultrarapid Optical Frequency-Domain Reflectometry Based Upon Dispersion-Induced Time Stretching: Principle and Applications". IEEE J. Sel. Top. Quantum Electron., vol. 18, no. 1, pp. 148-165, 2012.

[9] K. Goda, D. R. Solli, B. Jalali, "Real-time optical reflectometry enabled by amplified dispersive Fourier transformation," Appl. Phys. Lett, vol. 93, no. 3, pp. 031106/1- 031106/3, 2008.

[10] Yitang Dai, Jilong Li, Ziping Zhang, Feifei Yin, Wangzhe Li, and Kun $\mathrm{Xu}$, "Real-time frequency-to-time mapping based on spectrally-discrete chromatic dispersion," Opt. Express, vol. 25, no. 14, pp. 60-71, 2017.

[11] C. R. Fernandez-Pousa, J. Mora, H. Maestre and P. Corral, "Radiofrequency low-coherence interferometry," Opt. Lett., vol. 39, no. 12, pp. 3634-3637, 2014.

[12] J. Benítez and J. Mora, "Sensitivity Enhancement for Low-Coherence Interferometry," IEEE Photon. Technol. Lett., vol. 29, no. 20, pp. 17351738, 2017.

[13] J. Benítez, M. Bolea, and J. Mora, "Demonstration of multiplexed sensor system combining low coherence interferometry and microwave photonics," Opt. Express, vol. 25, no. 11, pp. 12182-12187, 2017.

[14] J. Mora, B. Ortega, A. Díez, J. L. Cruz, M. V. Andrés, J. Capmany, and D. Pastor, "Photonic Microwave Tunable Single-Bandpass Filter Based on a Mach-Zehnder Interferometer," J. Lightwave Technol., vol. 24, no. 7, pp. 2500-2509, 2006. 